\title{
Elderly condom use and perception: a barrier to family planning and mitigation of HIV/AIDS in high risk urban slums in Nigeria
}

\author{
K Odor
}

From International Conference on Prevention \& Infection Control (ICPIC 2011)

Geneva, Switzerland. 29 June - 2 July 2011

\section{Introduction / objectives}

As HIV/AIDS continues to pose a public health challenge Africa, the pandemic cuts across borders. It affects all the age groups including the geriatrics, despite engagement in risky sexual activities which increases HIV/AIDS infection. However, limited attention is paid to this population in mitigating the pandemic. This study therefore examined condom-use and perceived HIV/AIDS infection among geriatrics in Nigeria.

\section{Methods}

The study was cross-sectional in design. A multi-stage sampling procedure was used to select 400 -geriatrics. Pre-tested questionnaire developed, using information obtained from 10 Focus Group Discussion (FGD), was used to collect information. FGD data were analyzed thematically, while questionnaire data were analyzed using descriptive and statistically.

\section{Results}

Twenty-five percent of the participants had extra-marital sex since they attained elderly age. However, among this subgroup that had extra-marital sex, few (6.8\%) used a condom. More males (5.3\%) than females (1.5\%) used condom during the last extramarital sex. Low level of condom-use was attributed to condom not worthwhile (34.5\%) and opinion (50.0\%) condom not made for the elderly. Moreover, FGD participants viewed sex could not lead to pregnancy and majority $(60.3 \%)$ posited patronizing traditional healers and few $(10.3 \%)$ use of herbs/concussion could prevent HIV/AIDS. Similarly, non-condom use was due to confidence in traditional herbs, perceived to protect against STIs including HIV/ AIDS.

\section{Conclusion}

Engagement in risky activities among elderly is a growing HIV/AIDS challenge. Condom-use is misconstrued probably due to knowledge gap. Without urgent measures to enable them protect themselves, development efforts will be in jeopardy. Investing in geriatric SRH is cost-effective intervention in mitigating HIV/AIDS pandemic.

\section{Disclosure of interest}

None declared.

Published: 29 June 2011

doi:10.1186/1753-6561-5-S6-P290

Cite this article as: Odor: Elderly condom use and perception: a barrier to family planning and mitigation of HIV/AIDS in high risk urban slums in Nigeria. BMC Proceedings 2011 5(Suppl 6):P290. 